\title{
Reliable Quantification of EELS Spectra with a Simple Model-Based Approach
}

\section{G. Duscher, M.E. Hmielewski, and J.D.O. Oduor}

Dept. of Materials Science and Engineering, The University of Tennessee, Knoxville, TN 37996

Electron energy-loss spectroscopy in a TEM is one of the few tools that can obtain chemical composition with atomic column spatial resolution. This high spatial resolution is especially important for the characterization of interfaces and nanostructured materials. The error in chemical composition determination from electron energy-loss spectra are governed by background subtraction. Here we report a model based method in which we can reliably and reproducibly determine this background. The fit is checked visibly and mathematically. In contrast to the approach of Verbeeck et al.[1] the quantification relies on using the free atom part of the cross section only, making this a standartless analysis, which uses the conventional cross-sections only. Additionally, this method is capable to reliably determine the chemical composition even for overlapping edges. The method is established in an Excel spreadsheet, taking advantage of the non-linear solver within this program.

The method proposed by Verbeeck and van Aert is to calculate the cross sections including the near edge structure. But, the difficulty and subsequent lack of availability of these near edge structures are one reason why the so far most advanced quantification method of Verbeeck et al.[1][2] is not practical.

Steele et al. proposed a method to use least square fitting of the background and the cross-section simultaneously early on, but they proposed a fit for each ionization edge separately [3]. The here proposed method is also very close to the method as described by Leapman et al. [4], where reference spectra are fitted to an experemental spectrum to analyse these spectra. The difference of our method to Leapman's is that we can use the traditionaly used standard less cross sections.

The limitation of the method is clearly that spectra of thicker areas can only be analyzed successfully after single scattering analysis, because plasmon contributions to the edge will change the slope of the edge. The difference between the use of Hydrogenic and Hartree-Slater cross sections is not significant, because their slope at higher energies is similar.

This paper highlightsdifferent EELS investigations, each demonstrating quantitative analysis simplified with the QuantiFit spectrum analysis procedure. Quantitative determination of chemical composition of bulk $\mathrm{SrTiO}_{3}$, interfacial As , and $\mathrm{FeO}$ nanoparticle are examples we use to illustrate this new simple model based analysis which we named QuantiFit can aide in reliable quantification of experimental EELS spectra.

[1] J. Verbeeck and S. Van Aert. Ultramicroscopy, 101:207-224, 2004.

[2] J. Verbeeck, S. Van Aert, and G. Bertoni. Ultramicroscopy, 106:976-980, 2006.

[3] J.D. Steele and et. al. . Ultramicroscopy, 17:273-276, 1985.

[4] R. D. Leapman and C. R. Swyt. Ultramicroscopy, 26:393-404, 1988. 


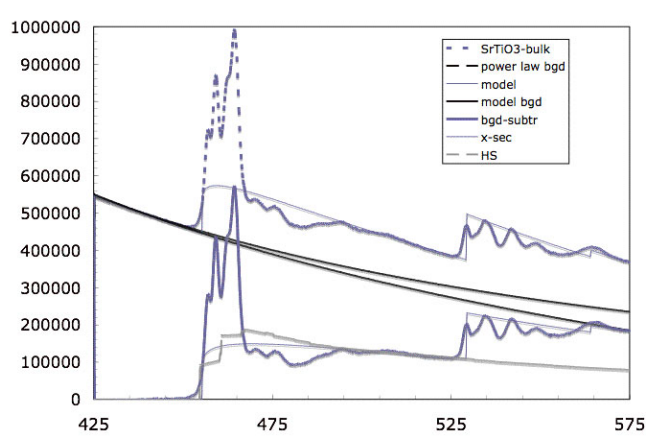

Figure 1: Quantification of a $\mathrm{SrTiO}_{3}$ bulk spectrum (fat dark gray, before and after background subtraction) with the Quantifit method. The fit is show as thin dark gray lines. The fit shows perfect stoichiometery with a reduced ${ }^{2}$ value of 13 . The black curves shows the backgrounds. The upper one is the power law background fitted to the pre edge slope and the lower one is the one modified by Quantifit routine.. Please note that the fit excluded $5 \mathrm{eV}$ before and $50 \mathrm{eV}$ after the edge onsets of each ionization edge (Ti- $\mathrm{L}_{2} 3$ at $455 \mathrm{eV}$ and $\mathrm{O}-\mathrm{K}$ at 530 $\mathrm{eV})$.

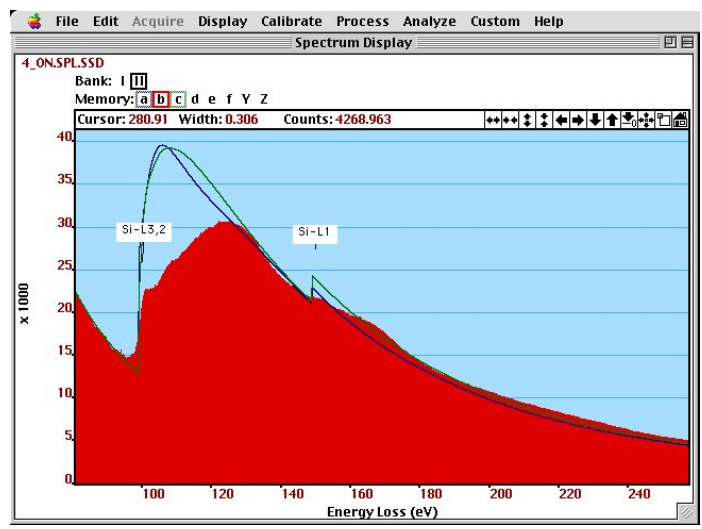

Figure 2: The shape of a Hydrogenic (green) and Hatree-Slater (blue) cross-section (with a background added) is compared to an experimental (SSD-corrected) spectrum of Si. 